\title{
A influência da atividade física na doença hepática gordurosa não alcoólica
}

The influence of physical activity in non-alcoholic fatty liver disease

La influencia de la actividad física en la enfermedad del hígado graso no alcohólico

Raphaella Maria Oliveira Pereira GOMES ${ }^{1}$

Victor Fernando Costa Macedo NORONHA ${ }^{\mathbf{1}}$

Gabriel Ponciano Santos de CARVALHO ${ }^{1}$

Ana Amélia Barreto FONTES ${ }^{1}$

Sônia Oliveira LIMA ${ }^{1}$

Joseane Barbosa de JESUS ${ }^{2}$

Mário Augusto Ferreira $\mathbf{C R U Z}^{3}$

Luis Guilherme Rosifini Alves REZENDE ${ }^{4}$ Josilda Ferreira $\mathbf{C R U Z}^{\mathbf{1}}$

${ }^{1}$ Universidade Tiradentes (UNIT), Aracaju-SE, Brasil

${ }^{2}$ Departamento de Educação Física da Universidade Federal de Sergipe (UFS), Aracaju-SE, Brasil

${ }^{3}$ Faculdade de Medicina de Ribeirão Preto da Universidade de São Paulo (FMRP-USP) 14049-900 Ribeirão Preto - SP, Brasil

${ }^{4}$ Médico Assistente do Programa de Cirurgia da Mão, Hospital das Clínicas da Faculdade de Medicina de Ribeirão Preto da Universidade de São Paulo, HC-FMRP-USP, Campus Monte Alegre, 14049-900 Ribeirão Preto - SP, Brasil

\section{Resumo}

O objetivo do presente trabalho foi associar a presença de esteatose hepática não alcoólica diagnosticada pela ultrassonografia (USG) com os níveis de atividade física. Estudo clínico, prospectivo, tipo survey, com dados coletados em um centro de referência em USG. A coleta de dados foi dividida em três etapas: aplicação de questionários, tendo destaque o Questionário Internacional de Atividade Física (IPAQ) em sua versão curta; aferição das medidas antropométricas e exame de USG abdominal. A análise estatística foi realizada através do software R, versão 3.6.1. O estudo foi aprovado pelo Comitê de Ética em Pesquisa. Foram analisados 119 pacientes, de ambos os sexos, com idade entre 18 e 70 anos e 06 atenderam aos critérios de exclusão. Demonstrou-se que $59,29 \%$ dos pacientes apresentavam o diagnóstico de esteatose hepática não alcoólica. Destes, 59,7\% eram sedentários. Concluiu-se que a inatividade física apresentou associação significativa com a presença da infiltração gordurosa no fígado.

Descritores: Fígado Gorduroso; Ultrassonografia; Estilo de Vida Sedentário; Obesidade.

\section{Abstract}

The purpose of the present study was to associate the presence of non-alcoholic hepatic steatosis diagnosed by ultrasound (USG) with the levels of physical activity. It was a clinical, prospective and survey type study, with data collected at an ultrasound reference center. The data collection was divided into three stages: application of questionnaires, with emphasis on the International Physical Activity Questionnaire (IPAQ), in its short version; measurements of anthropometric values and abdominal USG examination. The statistical analysis was performed using software R, version 3.6.1. The study was approved by the Research Ethics Committee. 119 patients of both sexes, aged between 18 and 70 years old, were analyzed, and 06 met the exclusion criteria. It was shown that $59.29 \%$ of patients had the diagnosis of non-alcoholic liver steatosis. Of these, $59.7 \%$ were sedentary. It was concluded that physical inactivity has a significant association with the presence of fatty infiltration in the liver.

Descriptors: Fatty Liver; Ultrasonography; Sedentary Lifestyle; Obesity.

\section{Resumen}

El objetivo del presente estudio fue asociar la presencia de esteatosis hepática no alcohólica diagnosticada por ecografía (USG) con los niveles de actividad física. Estudio clínico, prospectivo, tipo encuesta, con datos recopilados en un centro de referencia de la USG. La recolección de datos se dividió en tres etapas: aplicación de cuestionarios, destacando el Cuestionario Internacional de Actividad Física (IPAQ) en su versión corta; mediciones antropométricas y examen ecográfico abdominal. El análisis estadístico se realizó con el software R, versión 3.6.1. El estudio fue aprobado por el Comité de Ética en Investigación. Se analizaron 119 pacientes de ambos sexos, con edades comprendidas entre 18 y 70 años, y 06 cumplieron los criterios de exclusión. Se demostró que el 59,29\% de los pacientes tenían diagnóstico de esteatosis hepática no alcohólica. De estos, el 59,7\% eran sedentarios. Se concluyó que la inactividad física se asoció significativamente con la presencia de infiltración grasa en el hígado.

Descriptores: Hígado Graso; Ultrasonografía; Estilo de Vida Sedentario; Obesidad.

INTRODUÇÃO

A pandemia de sobrepeso e obesidade contribuiu para o aumento de complicações cardiovasculares e metabólicas. O acúmulo de gordura nos hepatócitos pode levar a um excesso de gordura hepática e associa-se ao desenvolvimento de doença hepática gordurosa não alcoólica ${ }^{1}$.

A Doença Hepática Gordurosa Não Alcoólica (DHGNA) compreende o espectro histopatológico de uma afecção no fígado que progride da esteatose para esteatohepatite não alcoólica (EHNA). É considerada uma doença multissistêmica que aumenta o risco de cirrose e carcinoma hepatocelular, além de contribuir para o desenvolvimento de diabetes mellitus tipo 2, doença cardiovascular e doença renal crônica. Histologicamente, a DHGNA é caracterizada por esteatose que afeta mais de $5 \%$ dos hepatócitos, e a esteatose com inflamação leve pode progredir para EHNA, com aumento da inflamação, lesão de hepatócitos e fibrose ${ }^{2}$.

Estima-se que a DHGNA substitua a hepatite viral como o principal fator para doença hepática terminal e transplante de fígado até $2023^{3}$. A sua prevalência global é estimada em $24,4 \%$ na população geral e fatores como ganho de peso, maior consumo de alimentos industrializados e processados, mudanças na dieta para uma tipicamente ocidental e estilo de vida sedentário podem contribuir para a tendência de crescimento da sua prevalência ${ }^{4}$. A maioria dos pacientes com DHGNA é obesa ou com sobrepeso e está na quarta à 
sexta década de vida. Esses pacientes geralmente são assintomáticos clinicamente, mas também podem apresentar hepatoesplenomegalia, fadiga, dispepsia e desconforto no quadrante superior direito. $\mathrm{Na}$ evolução para cirrose, os pacientes podem apresentar icterícia, ascite ou sangramento por varizes ${ }^{5}$.

A adiposidade, definida pelo índice de massa corporal (IMC), é um importante fator para desenvolvimento de DHGNA. O risco relacionado a adiposidade é predominantemente atribuído ao desenvolvimento de resistência à insulina $(R I)$ e disfunção do tecido adiposo nesses indivíduos ${ }^{2}$.

Estudos atuais sugerem que a DHGNA se desenvolve devido à deposição de lipídios e gorduras nos hepatócitos. Diversos fatores de risco desempenham um papel vital no acúmulo dessa gordura, como o tipo e o conteúdo da dieta, obesidade, síndrome metabólica (SM) e falta de atividade física. $O$ aumento da ingestão de carboidratos e proteínas animais está ligado ao aparecimento da DHGNA. A obesidade, outro fator de risco importante, perturba as vias oxidativas nos tecidos adiposos e esse estresse oxidativo cria condições desfavoráveis para 0 fígado, acarretando desenvolvimento e complicações da DHGNA. A inatividade física tem sido associada à deterioração e progressão da infiltração gordurosa hepática ${ }^{5}$.

A ultrassonografia (USG) abdominal é a modalidade de imagem mais usada para diagnóstico da DHGNA. A tomografia computadorizada (TC) e a ressonância magnética $(R M)$ são outros métodos de imagem não invasivos, entretanto, a biópsia hepática ainda permanece como padrão-ouro para a sua investigação ${ }^{5}$.

O gerenciamento de primeira linha da DHGNA é a modificação do estilo de vida, incluindo restrição alimentar e aumento da atividade física habitual ${ }^{3}$. O acúmulo de gordura hepática é positivamente associado ao tecido adiposo visceral, como também a fatores de risco cardiometabólicos independentes. Em adultos com ou sem DHGNA, o balanço energético negativo induzido pelo exercício, consistente com as diretrizes atuais, está associado a uma redução acentuada da gordura hepática ${ }^{6}$.

Existem evidências consideráveis que apoiam as associações de altos níveis de condicionamento físico e atividade física com diminuição da resistência à insulina e fatores de risco cardiometabólicos ${ }^{1}$. Tais hábitos podem prevenir o desenvolvimento e a progressão da DHGNA, através de diferentes mecanismos, como o aumento da energia gasta pelas contrações musculares e a estimulação da captação da glicose da circulação para os músculos ${ }^{7}$.

Uma questão associada à prática de atividade física é determinar qual dos seus tipos, aeróbica ou de alta resistência, tem maior benefício na DHGNA. Exercícios aeróbicos são altamente recomendados para melhorar marcadores de injúria hepática, como níveis de alanina aminotransferase (ALT) e aspartato aminotransferase (AST), além das características histológicas da DHGNA, que podem ser analisadas pelo seu escore de atividade $^{8}$.

O objetivo deste estudo foi associar a presença de esteatose hepática não alcoólica diagnosticada pela USG com os níveis de atividade física. Com isso, será possível traçar os impactos do estilo de vida no desenvolvimento da DHGNA, tendo em vista a sua base fisiopatológica, além de orientar intervenções na dieta e na prática de exercícios físicos, pontos fundamentais no tratamento de doenças metabólicas.

MATERIAL E MÉTODO

Estudo clínico, prospectivo, tipo survey. Os dados foram coletados em um centro de referência de ultrassonografia no município de Aracaju-SE, no período de abril/2019 a julho/2019, após aprovação do Comitê de Ética em Pesquisa da Universidade Tiradentes, pelo parecer 3251112, e seguiu as diretrizes e normas para estudo com seres humanos.

Os critérios de inclusão foram: adultos de ambos os sexos de 18 a 70 anos de idade, submetidos a USG abdominal de rotina, sempre realizada pelo mesmo examinador. Os critérios de exclusão foram: consumo de álcool $\geq$ $140 \mathrm{~g} / \mathrm{semana}$ nos homens e $\geq 70 \mathrm{~g} / \mathrm{semana}$ nas mulheres, portadores de neoplasias malignas primárias do fígado, portadores de doenças crônicas do fígado, portadores de deficiência física, portadores de deficiência cognitiva e pacientes em uso regular de drogas indutoras de esteatose hepática (esteroides, amiodarona e tamoxifeno).

O procedimento de coleta foi dividido em três etapas.

- Etapa 1

- Assinatura do TCLE;

- Preenchimento do questionário de saúde com informações sociodemográficas e de comorbidades, com as seguintes variáveis: nome, idade, data de nascimento, local de nascimento, profissão, uso de medicamentos, frequência de etilismo e comorbidades como 
doenças crônicas do fígado e deficiência física; - Aplicação do Questionário Internacional de Atividade Física (IPAQ), em sua versão curta validada por Matsudo et al. ${ }^{9}$, que conceitua e classifica os indivíduos em cinco categorias, de acordo com o consenso realizado entre 0 Centro de Estudos do Laboratório de Aptidão Física de São Caetano do Sul (CELAFISCS) e o Center for Disease Control (CDC) ${ }^{9}$.

- Muito ativo: aquele que cumpriu as recomendações:

$\checkmark$ VIGOROSA: $\geq 5$ dias/sem e $\geq 30$ minutos por sessão;

$\checkmark$ VIGOROSA: $\geq 3$ dias/sem e $\geq 20$ minutos por sessão + MODERADA e/ou CAMINHADA: $\geq 5$ dias/sem e $\geq 30$ minutos por sessão.

- Ativo: aquele que cumpriu as recomendações:

$\checkmark$ VIGOROSA: $\geq 3$ dias/sem e $\geq 20$ minutos por sessão;

$\checkmark$ MODERADA ou CAMINHADA: $\geq 5$ dias/sem e $\geq 30$ minutos por sessão;

$\checkmark$ Qualquer atividade somada: $\geq 5$ dias/sem e $\geq 150$ minutos/sem (caminhada + moderada + vigorosa;

- Irregularmente ativo: aquele que realiza atividade física, porém insuficiente para ser classificado como ativo, pois não cumpre as recomendações quanto à frequência ou duração. Para realizar essa classificação, foi somada a frequência e a duração dos diferentes tipos de atividades (caminhada + moderada + vigorosa). Este grupo foi dividido em dois subgrupos, de acordo com o cumprimento ou não de alguns dos critérios de recomendação:

$\checkmark$ IRREGULARMENTE ATIVO A: aquele que atinge pelo menos um dos critérios da recomendação quanto à frequência ou quanto à duração da atividade: i. Frequência: cinco dias / semana; ou ii. Duração: 150 min / semana;

$\checkmark$ IRREGULARMENTE ATIVO B: aquele que não atingiu nenhum dos critérios da recomendação quanto à frequência nem quanto à duração;

- Sedentário: aquele que não realizou nenhuma atividade física por pelo menos 10 minutos contínuos durante a semana;

Os pacientes foram então categorizados em cinco categorias: 1) muito ativo; 2) ativo; 3) irregularmente ativo $A ; 4$ ) irregularmente ativo $B$; sedentário.

O cálculo do consumo etílico pela fórmula: dose em $\mathrm{ml} x$ teor alcoólico x 0,8 / 100, onde o teor alcoólico das bebidas é conhecido, a saber: cerveja 4 , vinho 12 , conhaque 40 , rum 40 , uísque 43 , pinga $46^{10}$.

\section{- Etapa 2}

Realizada aferição das medidas antropométricas: peso, altura e circunferência da cintura. Para o peso corpóreo, foi utilizado uma balança portátil Techline® e, para isso o paciente permaneceu em pé, descalço, no centro da balança, com o peso distribuído em ambos os pés. A aferição da altura foi feita por meio de um estadiômetro portátil da marca Filizola®, com a leitura realizada no milímetro (mm) mais próximo, tendo o paciente se posicionado descalço, verticalmente com os braços estendidos ao longo do corpo, ombros relaxados, com os calcanhares juntos e a cabeça posicionada. Os dados de peso e altura foram utilizados para o cálculo do IMC pelo índice de Quetelet: razão entre o peso corpóreo em quilograma e altura em metro ao quadrado. A CC foi medida com o paciente em pé, utilizando uma fita métrica inelástica ao nível do ponto médio entre a última costela e a crista ilíaca ${ }^{11}$.

\section{- Etapa 03}

Realizado o exame de ultrassonografia abdominal modo B com transdutor convexo, dinâmico, com formação da imagem contínua e automática, de frequência de $3,75 \mathrm{MHz}$. Os pacientes se apresentaram com preparo adequado, com jejum de no mínimo 6 horas e uso de antiflatulentos. A esteatose hepática não alcoólica foi classificada em graus de acordo com estudo realizado em 2002 (12):

$\checkmark$ Grau 0: Ecogenicidade normal;

$\checkmark$ Grau 1: Esteatose Leve, com visualização de ecos finos do parênquima hepático, visualização normal do diafragma e dos vasos intrahepáticos;

$\checkmark$ Grau 2: Esteatose moderada, com aumento difuso nos ecos finos, visualização prejudicada dos vasos intrahepáticos e do diafragma;

$\checkmark$ Grau 3: Esteatose severa, com um aumento importante dos ecos finos, visualização prejudicada ou ausente dos vasos intra-hepáticos.

Os dados foram compilados no programa Microsoft Office Excel e foram obtidas variáveis qualitativas e quantitativas. Para as variáveis qualitativas, a análise descritiva procedeu com a categorização dos dados e obtenção das respectivas frequências e percentuais e o cálculo da média, desvio padrão, mínimo e máximo para as variáveis quantitativas.

Para verificar a relação entre as variáveis qualitativas "presença ou não da esteatose hepática" e "níveis de atividade física", foi utilizado o teste Qui-quadrado de Pearson. 
No teste de hipótese realizado, a conclusão foi obtida através da interpretação do p-valor. Adotando um nível de significância de $5 \%$, sempre que o $p$-valor calculado for menor que 0,05 houve associação entre as variáveis analisadas. $O$ software utilizado foi o $R$, versão 3.6.1.

\section{RESULTADOS}

Foram avaliados 119 pacientes, sendo que, destes, 06 atenderam aos critérios de exclusão, totalizando uma amostra final de 113. Do total de pacientes envolvidos neste estudo (Tabela 1), demonstrou-se que $59,29 \%$ apresentavam 0 diagnóstico de esteatose hepática não alcoólica, onde $42(67,2 \%)$ eram do sexo feminino e 25 (37,3\%), do masculino. Quanto à prática de atividade física, a maioria dos pacientes diagnosticados com infiltração gordurosa hepática foram classificados como nível 5 ( $n=40 ; 59,7 \%)$, ou seja, sedentários. A idade média dos portadores de esteatose hepática não alcoólica foi de 46,9 anos e o peso variou entre 49,4 e 126,6 kg, sendo o peso médio $82,9 \mathrm{Kg}$.

O IMC médio dos pacientes acometidos pela gordura hepática foi $30,8 \mathrm{~kg} / \mathrm{m} 2$, enquanto o dos pacientes que não apresentaram alterações ultrassonográficas de esteatose no fígado foi de $26,2 \mathrm{~kg} / \mathrm{m} 2$.

Ainda no que se refere aos pacientes que não possuíam a esteatose hepática não alcoólica, o sexo predominante foi o feminino $(76,1 \% ; n=35)$ e a média de idade foi 40,7 anos. A maioria deles se encaixava em algum grupo considerado ativo pelo questionário IPAQ, totalizando $52,2 \%$.

Tabela 1: Variáveis dos pacientes analisados em relação à presença ou não de esteatose hepática não alcoólica pela ultrassonografia abdominal

\begin{tabular}{r|c|l|l|l}
\multicolumn{5}{c}{ ESTEATOSE SIM } \\
\hline Variáveis & n & & \% & \\
\hline Sexo & & & & \\
\hline Masculino & 25 & & 37,3 & \\
\hline Feminino & 42 & & 62,7 & \\
\hline Idade (anos) & 25,0 & 70,0 & 46,9 & 12,1 \\
\hline Peso(kg) & 49,4 & 126,6 & 82,9 & 17,4 \\
\hline Altura(m) & 1,4 & 1,9 & 1,6 & 0,1 \\
\hline $\begin{array}{r}\text { IMC } \\
\text { (kg/m2) }\end{array}$ & 20,2 & 40,8 & 30,8 & 4,8 \\
\hline \multicolumn{5}{|c}{ ESTEATOSE NÃO } \\
\hline
\end{tabular}

\begin{tabular}{|c|c|c|c|c|}
\hline \multicolumn{5}{|l|}{ Variáveis } \\
\hline & $\mathbf{n}$ & & $\%$ & \\
\hline \multicolumn{5}{|l|}{ Sexo } \\
\hline Masculino & 11 & & 23,9 & \\
\hline \multirow[t]{2}{*}{ Feminino } & 35 & & $\begin{array}{l}23,9 \\
76,1\end{array}$ & \\
\hline & Min & Max & Média & DP \\
\hline Idade (anos) & 16,0 & 70,0 & 40,7 & 14,9 \\
\hline Peso(kg) & 44,4 & 107,2 & 67,4 & 13,6 \\
\hline Altura(m) & 1,5 & 2,0 & 1,6 & 0,1 \\
\hline $\begin{array}{r}\text { IMC } \\
(\mathrm{kg} / \mathrm{m} 2)\end{array}$ & 17,7 & 36,2 & 26,2 & 4,4 \\
\hline
\end{tabular}

A Tabela 2 evidenciou uma associação estatisticamente significativa entre o nível de atividade física e a presença ou não de esteatose hepática não alcoólica $(p=0,038)$.

Tabela 2. Nível de atividade física em relação a presença ou não de esteatose hepática não alcoólica diagnosticada pela ultrassonografia abdominal

\begin{tabular}{|c|c|c|c|c|c|}
\hline \multirow{3}{*}{$\begin{array}{l}\text { ATIVIDADE } \\
\text { FÍSICA }\end{array}$} & \multicolumn{5}{|c|}{ ESTEATOSE } \\
\hline & \multicolumn{2}{|r|}{ SIM } & \multicolumn{2}{|c|}{ NÃO } & \multirow[b]{2}{*}{$\boldsymbol{p}$} \\
\hline & $\mathbf{n}$ & $\%$ & $\mathbf{n}$ & $\%$ & \\
\hline $\mathbf{1}$ & 4 & 6,0 & 9 & 19.6 & \multirow{5}{*}{0,038} \\
\hline 2 & 7 & 10,4 & 3 & 6,5 & \\
\hline 3 & 8 & 11,9 & 7 & 15,2 & \\
\hline 4 & 8 & 11,9 & 5 & 10,9 & \\
\hline 5 & 40 & 59,7 & 22 & 47,78 & \\
\hline \multicolumn{6}{|c|}{$\begin{array}{l}\text { Legenda: } 1 \text { : muito ativo; } 2 \text { : ativo; 3: irregularmente ativo A; } 4 \text { : irregularmente ativo B; } \\
\text { sedentário; n: frequência absoluta; \%: frequência relativa; } p \text { : significância estatística. } \\
\text { Fonte: Dados da pesquisa }\end{array}$} \\
\hline DISCUSSÃO & & & & & \\
\hline
\end{tabular}

Com a crescente prevalência de doenças crônicas não transmissíveis em todo o mundo, a DHGNA tornou-se um novo desafio para a saúde pública ${ }^{5}$. Esta é uma doença multissistêmica, caracterizada pelo armazenamento de gordura nos hepatócitos e sua evolução para esteatose não causada pelo consumo excessivo de álcool. A atividade física como modificadora do estilo de vida desempenha um papel importante na prevenção da DHGNA ${ }^{3}$. Isso se deve não só ao seu papel na perda de peso, mas também a sua capacidade de melhorar a função hepática, independentemente do emagrecimento ${ }^{13}$.

A frequência de atividade física tem influência nos indivíduos com DHGNA, mostrando que esta afecção se apresentou significativamente menor nos que praticavam exercícios físicos e que aproximadamente metade dos indivíduos nunca optou por algum tipo de treino ${ }^{5}$. Em concordância, a presente análise constatou que, enquanto $52,2 \%$ dos pacientes que não apresentaram esteatose hepática não alcoólica se exercitavam, apenas $40,3 \%$ dos portadores desta afecção eram ativos fisicamente.

Constou-se que indivíduos portadores de DHGNA passam a maior parte do tempo em hábitos sedentários e com menos atividade física diariamente, em comparação com indivíduos não acometidos ${ }^{14}$. Não obstante, a incidência de DHGNA aumenta entre indivíduos com um estilo de vida sedentário ${ }^{15}$. Da mesma maneira, o presente estudo constatou que a doença é mais prevalente naqueles que não realizam nenhuma atividade física por pelo menos 10 minutos contínuos durante a semana. Dos 67 pacientes diagnosticados com DHGNA, $59,7 \%$ assumiram ter um estilo de vida inativo.

A prática de atividade física pode reduzir significativamente o conteúdo de gordura 
hepática do paciente com DHGNA ${ }^{3}$. Congruentemente, esta pesquisa mostrou que apenas $5,9 \%$ dos pacientes com fígado gorduroso foram considerados "muito ativos" pelo questionário IPAQ, visto que se exercitam por pelo menos três dias por semana, realizando atividades vigorosas e moderadas.

A prevalência da esteatose hepática não alcoólica aumenta como consequência do sedentarismo e das maiores taxas de obesidade $^{16}$. Ao analisar o IMC dos pacientes portadores de DHGNA, este trabalho corroborou tal dado, ao obter um valor médio de 30,8 $\mathrm{kg} / \mathrm{m}^{2}$, que corresponde a um número maior que o recomendado pela Organização Mundial da Saúde (OMS), que considera normais valores $\geq$ $18,5 \mathrm{~kg} / \mathrm{m}^{2} \mathrm{e}<25 \mathrm{~kg} / \mathrm{m}^{2}$.

Exercícios aeróbicos mostraram ser efetivos na redução da gordura no fígado ${ }^{17}$. Em adolescentes obesos, a prevalência da DHGNA apresentou menor nos grupos não sedentários $^{18}$. O exercício físico rigoroso reduz - peso corporal, aumenta a força muscular, melhora o perfil lipídico e promove melhor controle dos níveis glicêmicos ${ }^{19}$. Além disso, reduz a pressão arterial, melhora a condição cardiovascular e beneficia a saúde psicossocial e a autoestima do indivíduo ${ }^{20}$. No presente estudo, a inatividade física esteve relacionada à maior presença de esteatose hepática não alcoólica, logo, evidencia-se que exercícios físicos influenciam na saúde do indivíduo sendo um fator importante de prevenção da DHGNA.

\section{CONCLUSÃO}

A presente pesquisa concluiu que a inatividade física apresentou associação significativa com a presença de esteatose hepática não alcoólica. Realizou-se um estudo observacional, evidenciando o sedentarismo como fator de desenvolvimento da infiltração gordurosa hepática. Entretanto, como estes os pacientes foram analisados em um único momento, é importante que estudos longitudinais possam confirmar os benefícios da atividade física como fator efetivo no melhor prognóstico da DHGNA.

\section{REFERÊNCIAS}

1. Medrano $\mathrm{M}$, Arenaza $\mathrm{L}$, Migueles $\mathrm{JH}$, Rodríguez-Vigil B, Ruiz JR, Labayen I. Associations of physical activity and fitness with hepatic steatosis, liver enzymes, and insulin resistance in children with overweight/obesity. Pediatr Diabetes. 2020;21(4):565-74.

2. Shaunak M, Byrne CD, Davis $N$, Afolabi $P$, Faust SN, Davies JH. Non-alcoholic fatty liver disease and childhood obesity. Arch Dis Child. 2021;106(1):3-8.
3. Wang ST, Zheng J, Peng HW, Cai XL, Pan XT, Li HQ, Hong QZ, Peng XE. Physical activity intervention for non-diabetic patients with nonalcoholic fatty liver disease: a meta-analysis of randomized controlled trials. BMC Gastroenterol. 2020;20(1):66.

4. Yari Z, Cheraghpour M, Aghamohammadi V, Alipour M, Ghanei N, Hekmatdoost A. Energydense nutrient-poor snacks and risk of nonalcoholic fattyliver disease: a case-control study in Iran. BMC Res Notes. 2020;13(1):221.

5. Tahir F, Majid Z, Majid B, Ahmed J, Zaman A, Tariq $M$ et al. Anthropometric Measurements and Lifestyle Characteristics of Individuals with Non-alcoholic Fatty Liver Disease. Cureus. 2020;12(2):e7016.

6. Ross R, Soni S, Houle SA. Negative Energy Balance Induced by Exercise or Diet: Effects on Visceral Adipose Tissue and Liver Fat. Nutrients. 2020;12(4):891.

7. Stevanović J, Beleza J, Coxito P, Ascensão A, Magalhães J. Physical exercise and liver "fitness": Role of mitochondrial function and epigenetics-related mechanisms in nonalcoholic fatty liver disease. Mol Metab. 2020;32:1-14.

8. Kwak MS, Kim D. Non-alcoholic fatty liver disease and lifestyle modifications, focusing on physical activity. Korean J Intern Med. 2018;33(1):64-74.

9. Matsudo SM, Matsudo VR, Araújo T, Andrade D, Andrade E, Oliveira L et al. Nível de atividade física da população do Estado de São Paulo: análise de acordo com o gênero, idade, nível socioeconômico, distribuição geográfica e de conhecimento. Rev. Bras. Ciên. e Mov. 2002;10(4):41-50

10. Mincis M, Mincis R. Álcool e o fígado. GED gastroenterol endosc dig. 2011;30(4):152-62.

11. Lohman TG, Caballero B, Himes JH, Davis CE, Stewart D, Houtkooper $L$ et al. Estimation of body fat from anthropometry and bioelectrical impedance in Native American children. Int $\mathrm{J}$ Obes Relat Metab Disord. 2000;24(8):982-28.

12. Saadeh S, Younossi ZM, Remer EM, Gramlich $\mathrm{T}$, Ong JP, Hurley $\mathrm{M}$ et al. The utility of radiological imaging in nonalcoholic fatty liver disease. Gastroenterology.2002;123(3):745-50.

13. Ahmed IA, Mikail MA, Mustafa MR, Ibrahim M, Othman R. Lifestyle interventions for nonalcoholic fatty liver disease. Saudi J Biol Sci. 2019;26(7):1519-24.

14. Kenneally S, Sier JH, Moore JB. Efficacy of dietary and physical activity intervention in nonalcoholic fatty liver disease: a systematic review. BMJ Open Gastroenterol. 2017;4(1): e000139.

15. Gerber L, Otgonsuren M, Mishra A, Escheik C, Birerdinc A, Stepanova M et al. Non-alcoholic fatty liver disease (NAFLD) is associated with low level of physical activity: a population-based study. Aliment Pharmacol Ther. 
2012;36(8):772-81.

16. Yu R, Shi Q, Liu L, Chen L. Relationship of sarcopenia with steatohepatitis and advanced liver fibrosis in non-alcoholic fatty liver disease: a meta-analysis. BMC Gastroenterol. 2018; 18(1):51.

17. Bacchi E, Negri C, Targher G, Faccioli N, Lanza M, Zoppini G, Zanolin E, Schena F, Bonora E, Moghetti $P$. Both resistance training and aerobic training reduce hepatic fat content in type 2 diabetic subjects with nonalcoholic fatty liver disease (the RAED2 Randomized Trial). Hepatology. 2013;58(4):1287-95.

18. Dâmaso AR, Tock L., Tufik S, Prado WL, Stella SG, Fisberg $M$ et al. Tratamento multidisciplinar reduz o tecido adiposo, visceral, leptina, grelina e a prevalência de esteatose hepática não alcoólica (NAFLD) em adolescentes obesos. Rev Bras Med Esporte. 2006;12(5): 263-67.

19. Pinto CG iulian. de S, Marega M, Carvalho JAM alu. de, Carmona FG ambett., Lopes CE duard. F, Ceschini FL ui., et al. Physical activity as a protective factor for development of nonalcoholic fatty liver in men. Einstein. 2015;13(1):34-40.

20. Marçal DFS, Alexandrino EG, Cortez LER, Bennemann RM. Efeitos do exercício físico sobre diabetes mellitus tipo 1: Uma revisão sistemática de ensaios clínicos e randomizados. J Phys Educ. 2018;29:e2917.

\section{CONFLITO DE INTERESSES}

Os autores declaram não haver conflitos de interesse

\section{AUTOR PARA CORRESPONDÊNCIA}

\section{Raphaella Maria Oliveira Pereira Gomes}

Rua Euclides Góis 1297 Coroa do Meio

Tel: 79 99961-4677

E-mail: raphaella.ogomes@gmail.com 